\title{
FREQUENCY OF DENGUE INFECTION IN FEBRILE PATIENTS ATTENDED DHAKA MEDICAL COLLEGE HOSPITAL DURING JANUARY TO DECEMBER, 2018
}

\author{
SULTANA N ${ }^{1}$, FATEMA N ${ }^{2}$, HOSSAIN MZ ${ }^{3}$, RAHMAN MA ${ }^{4}$, NEHAR N $^{5}$, YEASMIN MM ${ }^{6}$, \\ SHARMIN R ${ }^{7}$, SWEETY AA ${ }^{8}$, PERVIN $\mathrm{M}^{9}$
}

\begin{abstract}
:
Introduction: Dengue is a major public health concern in our country. The alarming thing is that the seasonal trend of dengue infection is changing with time in Bangladesh due to climate change and unplanned urbanization. Our study was conducted to determine the frequency of dengue virus infection among the febrile patients in 2018 at Dhaka Medical College Hospital $(D M C H)$ and to observe the seasonal trend.

Materials and Methods: The study was carried out on 899 febrile patients attended in DMCH from January to December, 2018. Whole blood samples were collected and sera were tested for dengue NS1 antigen and anti-dengue IgM antibodies using commercial test kits (NS1 by OMC Healthcare (Pvt.) Ltd \& IgM antibody by Omega Diagnostics Ltd.), respectively. All negative dengue cases were tested for anti Chikungunya antibody to exclude chikungunya.

Results: Of the total 899 febrile patients, 350(38.93\%) were positive for Dengue infection. Out of them 264(75.43\%) were positive for NS1, 82 (23.43\%) were positive for IgM and $4(1.14 \%)$ were positive for dengue NS1antigen + anti dengue IgM antibody. More than 50\% patients belonged to age group 15-29 years. Males were predominant. More than 60\% cases were detected in the post monsoon season.

Conclusion: Highest dengue cases were detected in this year in comparison to the previous years probably due to re-emergence of DEN-3 serotype. Due to pattern of climate change, seasonal trend of dengue infection was not maintained. Moreover, unplanned urbanization and poor solid waste management have worsened the situation more.
\end{abstract}

Key words: Dengue Fever, Dengue NS1 antigen

J Dhaka Med Coll. 2019; 28(1) : 105-111

\section{Introduction}

Dengue is a common communicable arthropodborne viral disease and one of the most common causes of febrile illness in Bangladesh ${ }^{1}$. There are five antigenically distinct serotypes DEN-1, DEN-2, DEN-3, DEN-4 and DEN-5, of dengue viruses transmitted between humans by infected mosquitoes ${ }^{2}$. All of them are responsible for causing disease found in the tropical and subtropical regions worldwide ${ }^{3}$.
Infection with one serotype is alleged to confer lifelong serotype-speciûc immunity ${ }^{4}$. But preexisting antibody cannot confer protection against a different serotype. Consequently secondary infection with heterogeneous type is frequently associated with severe clinical manifestations e.g. dengue hemorrhagic fever/ dengue shock syndrome (DHF/DSS) which is considered to be linked with Antibody dependent enhancement ${ }^{5}$. The disease is

1. Dr. Nusrat Sultana, Assistant Professor, Department of Virology, Dhaka Medical College

2. Dr. Nusrat Fatema, Lecturer, Department of Virology, Dhaka Medical College

3. Dr. Mohammad Zaid Hossain, Associate Professor, Department of Medicine, Dhaka Medical College, Dhaka

4. Dr. Md Anisur Rahman, Lecturer, Department of Virology, Dhaka Medical College

5. Dr. Naznin Nehar, Assistant Professor, Department of Microbiology, Jashore Medical College

6. Dr. Mst. Marufa Yeasmin, Assistant Professor, Department of Microbiology, Faridpur Medical College

7. Dr. Rabeya Sharmin, Assistant Professor, Department of Virology, Dhaka Medical College

8. Dr. Afroza Akbar Sweety, Assistant Professor, Department of Virology, Dhaka Medical College

9. Dr. Monira Pervin, Associate Professor, Department of Virology, Dhaka Medical College

Correspondence: Dr. Nusrat Sultana, Assistant Professor, Department of Virology, Dhaka Medical College, Mobile no. 01715006313, Email address: sultananusrat78@yahoo.co

Received: 10 February 2019

Revision: 02 March 2019

Accepted: 30 Mach 2019 
characterized by high grade fever with headache, retro-orbital pain, low back pain, skin rash, muscle or joint pain and bleeding manifestations ${ }^{6}$.

Dengue is an enveloped virus with single strand positive sense RNA genome encoding three structural proteins and seven nonstructural proteins (NS1, NS2A, NS2B, NS3, NS4A, NS4B and NS5) ${ }^{7}$. It is a Flavivirus belongs to Flaviviridae family transmitted mainly via species of Aedes aegypti and, as well by A. albopictus mosquitoes 8 . Due to global warming, climate change, civilization and urban to rural distribution of mosquitoes intensify the dengue virus infection as one of the neglected tropical diseases (NTDs) ${ }^{8}$.

Most subtropical and tropical regions around the world where Aedes vectors exist are endemic for Dengue outbreak. The global prevalence of dengue virus infection has been dramatically increased in the recent past and its epidemiological pattern is also changing $9,10,11$. In Bangladesh, the first Dengue cases were reported in 1964 and the outbreak was renowned as 'Dacca Fever'12. Thereafter, Dengue was reported in 1999 and in 2000. There was an outburst in 2000 and 5,551 dengue cases and 93 dengue-related deaths were recorded ${ }^{13,14}$. From then onwards, Dengue cases have been reported in small or large scales in every year. In Bangladesh, Dengue outbreak is mainly prevalent following the yearly monsoon rains from June to late September. Additionally, their tropical monsoon climates with high humidity where $A$. aegypti populations are more stable are aiding the incidence of DENV infection throughout a large period of the year ${ }^{15,16,17,18}$.

There are four clinical entities of Dengue virus infection including undifferentiated fever (UF), Dengue fever (DF), Dengue hemorrhagic fever (DHF) and Dengue Shock Syndrome (DSS) [9]. Early detection of Dengue cases is important to control its endemicity and it can be achieved by routinely performed serological tests of clinically suspected dengue patients.

Dengue virus infection is now recognized as an emerging threat to public health in Bangladesh.
The current study was undertaken to determine the occurrence of Dengue virus infection in febrile patients attending $\mathrm{DMCH}$ using wellestablished serological method. The data presented provide valuable information regarding current prevalence of the disease in Bangladesh and the present seasonal trend of dengue infection.

\section{Materials and Methods}

This cross-sectional study was carried out at Dhaka Medical College, a tertiary care teaching hospital at Dhaka from January 2018 to December 2018. A total of 899 Blood samples were received from all febrile patients who were referred to the Department of Virology, DMC irrespective of age and sex. Demographic details and clinical history were obtained and recorded.

Sera were separated and preserved at 4ÚC till the time of testing. Sera of patients with illness 5 days or less were tested for non-structural protein 1 (NS1) antigen by using ImmunoChromatographic Test (ICT), the commercially available rapid dengue diagnostic kit "In Bios" (In Bios International,Inc., USA). Sera of patients with illness 5 or more than 5 days were tested by dengue IgM antibody capture ELISA (MAC ELISA) for IgM antibody detection as per kit insert.

Clinical data were obtained by interviewing the patients or their attendants, entered into the preformatted data collection sheet. After taking all aseptic precaution $5 \mathrm{ml}$ of venous blood was collected from each patient and transferred into a properly labeled sterile clot activated tube. Serum sample was collected in labeled eppendorf tubes within 2-3 hours of blood collection by centrifugation of the blood sample and after proper inspection of sample that there was no haemolysis. The serum samples were preserved in a $-20^{\circ} \mathrm{C}$ fridge until the testing for NS1 antigen by Immuno chromatographic test (ICT) and IgM dengue antibody by ELISA method according to the instruction of the manufacturer. Dengue NS1 Ag kit (OMC Healthcare (Pvt.) Ltd, Canada) was used for NS1 and anti-dengue IgM (Omega Diagnostics Ltd, UK) kit was used to perform tests in patient's samples. 
According to our case definition, dengue positive cases diagnosed by NS1antigen and or antidengue IgM ELISA positive tests. NS1 and antidengue IgM ELISA negative cases were tested to detect chikungunya infection by anti chikungunya antibody test.

\section{Result:}

A total of 899 febrile patients were enrolled in this study. Among them 623 (69.3\%) and 276 $(30.7 \%)$ were male and female, respectively with the age $26.37 \pm 11.66$ years. Most of the patients irrespective of sex belonged to the age group $15-29$ years which is $65.41 \%$. The total duration of fever was $4.66 \pm 4.47$ days. Among the febrile patients $718(80 \%)$ and $181(20 \%)$ were from outdoor and indoor, respectively (Table-1).

Among the febrile patients, dengue was detected in $350(38.93 \%)$ cases (Figure 1). Among the males, $38.52 \%$ were affected, whereas, the rate is higher in females which is $39.86 \% .72 .57 \%$ Dengue cases are found mostly in 15-29 years age group. The overall age of the patients were $25 \pm 9.24$ years irrespective of age and sex. The duration of fever of these patients was $4.014 \pm 2.21$ years. Most of the patients were from outdoor $(80 \%)$.

The distribution of clinical manifestations in dengue cases is given in Table 2. Besides fever, common symptoms were headache $(61.14 \%)$, Myalgia (44\%), arthralgia (23.14), retro-orbital pain $(20.29 \%)$ and gastrointestinal symptoms (32.86\%). Other symptoms like loose motion, sub-conjunctival haemorrhages, rash were present in lower frequencies.

Out of 350 dengue patients, 264(75.43\%) were positive for NS1 antigen, 82(23.43\%) patients were positive for anti dengue IgM antibody and $4(1.14 \%)$ patients were positive for both NS1 and anti dengue IgM antibody shown in Shown in Figure 1.

In the present study, first dengue case was detected in the month of May and continued till December. The dengue cases began to rise from July and maximum cases were found in September and November with the frequency of $105(30 \%)$ and $112(32 \%)$, respectively. There was sudden drop of dengue cases in the month of October (figure 4).

\section{Table-I}

Demographic Profile and dengue cases in study participants.

\begin{tabular}{lcr}
\hline Characteristic & $\begin{array}{c}\text { Febrile } \\
\text { patients } \\
\mathrm{n}=899\end{array}$ & $\begin{array}{c}\text { Dengue } \\
\text { cases } \\
\mathrm{n}=350\end{array}$ \\
\hline $\begin{array}{l}\text { Age (years) } \\
\text { Mean } \pm \text { SD }\end{array}$ & $26.37 \pm 11.66$ & $25 \pm 9.24$ \\
Sex & $623(69.3)$ & $240(38.52)$ \\
Male & $276(30.7)$ & $110(39.86)$ \\
Female & $4.66 \pm 4.47$ & $4.014 \pm 2.21$ \\
$\begin{array}{l}\text { Duration of fever } \\
\text { (days)Mean } \pm \text { SD }\end{array}$ & & \\
Distribution of patients & & \\
Outdoor & $718(80)$ & $338(96.56)$ \\
Indoor & $181(20)$ & $12(3.44)$ \\
\hline
\end{tabular}

All the numbers in the parentheses is the percentage.

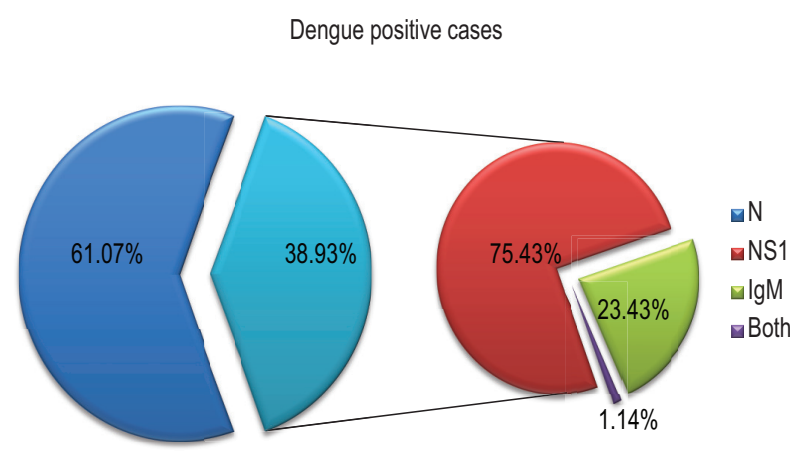

Fig.-1: A Pie of the Pie chart depicting dengue positive cases detected by NS1, anti dengue IgM and both tests.

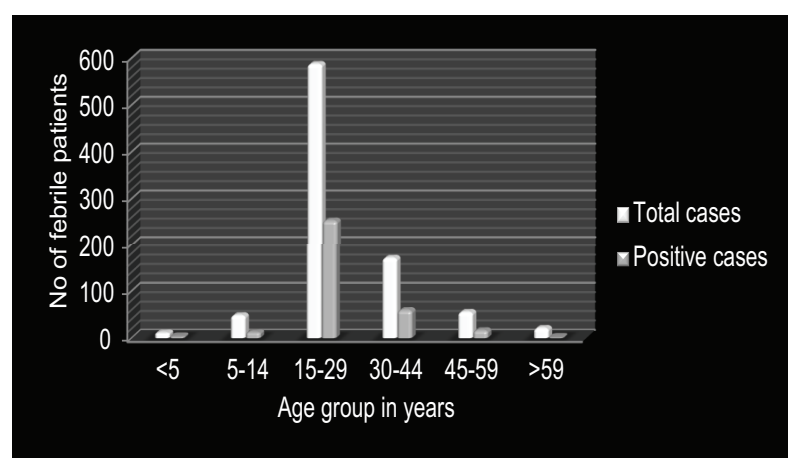

Fig.-2: A bar chart comparing the age distribution of the febrile patients and dengue positive cases. 


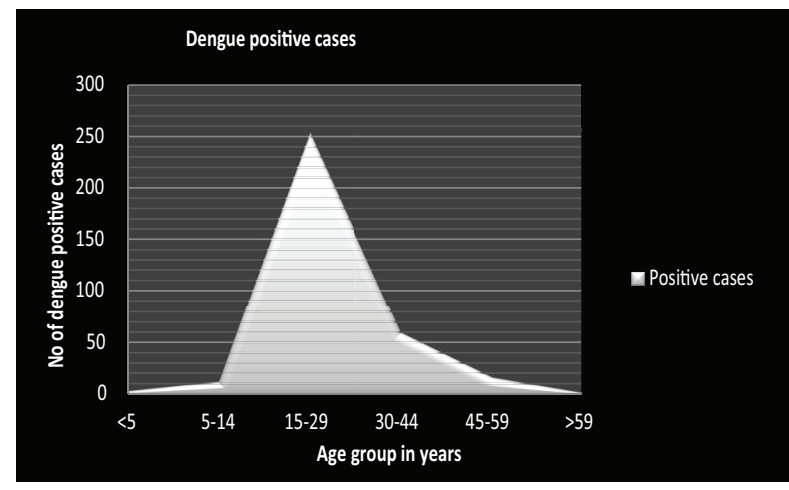

Fig.-3: Frequency of dengue cases among different age groups.

Table-II

Clinical Presentations observed among Dengue cases $(n=350)$

\begin{tabular}{lc}
\hline Clinical features & $\mathrm{n}(\%)$ \\
\hline Fever & $350(100)$ \\
Headache & $214(61.14)$ \\
Arthralgia & $81(23.14)$ \\
Retro-orbital pain & $71(20.29)$ \\
Myalgia & $154(44)$ \\
Low back pain & $11(3.14)$ \\
Gastro-intestinal symptoms & $115(32.86)$ \\
Rash & $21(6)$ \\
Loose motion & $18(5.14)$ \\
\hline
\end{tabular}

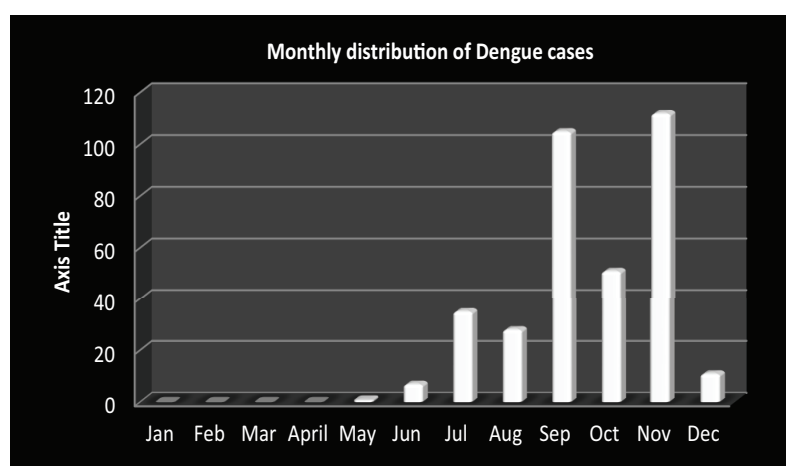

Fig.-4: Monthly frequency of dengue cases in the year 2018.

\section{Discussion:}

Overall incidences of dengue fever along with explosive infection outbreaks have been progressively increasing in frequency over the last several years, making it a major contemporary public health concern. In Bangladesh, persistent circulation of four dengue virus serotypes is responsible for frequent epidemics (http://apps.who.int/ disasters/repo/13755. pdf). During the period between January-December, 2018, blood samples were collected from a total of 899 febrile patients attending $\mathrm{DMCH}$ within Dhaka metropolitan city, upon being referred to the Virology Laboratory of Dhaka Medical College. Patients with fever durations occurring d" 5 days were tested for only Dengue NS1 while those extending $>5$ days, for both dengue NS1 and anti-dengue IgM. The total positive cases of dengue found were 350 (38.93\%). Among these cases, NS1 positive, anti-dengue IgM positive and NS1+ anti-dengue IgM positive test frequencies were at $264(75.43 \%), 82(23.43 \%)$ and $4(1.14 \%)$, respectively. Chikungunya infection was excluded from all the dengue negative cases by anti-chikungunya IgM screens.

This remarkably increased frequency has become a matter of great concern. By analyzing the genetic data of the previous years, DEN 3 was determined to be the predominant serotype until 2002, after which DEN3 has been replaced by DEN1 and DEN2. Because DENV-1 and DENV-2 were in circulation for over a decade prior, a large portion of the population of Bangladesh may have already developed immunity towards these serotypes. Yet, the risk of developing severe dengue infection by DENV 3 and DENV 4 could not hastily be ignored either, as these serotypes are still known to be in circulation ${ }^{18}$. Discovery of the highest proportion of total dengue cases occurring in the year 2018 compelled our researchers to consider serotypes other than DENV 1 and DENV2. Therefore, a genetic study was prompted, as conducted by IEDCR aiding to identify larger than expected proportions of DENV3 as a major contributor ${ }^{19}$.

Male patients were the predominant sex in our study with over twice as many participants as compared to females. However, the occurrence of dengue virus infection was not found to be significantly different among the sexes. In a 
patriarchal society such as Bangladesh, female welfare is often down played in accordance to socioeconomic perspectives. In contrast, as the main bread-winners, male fitness is often prioritised by family members due to their dominant role as part of the economic workforce. Therefore, it is a common belief that they should remain healthy in order to continue their jobs, uninterrupted. In our study, the majority of febrile patients belonged to the 1529 years age-range category with $>50 \%$ showing dengue positivity. The possible reasoning for this is that this age group consists mostly of high-school to university level students. As such, it is expected that they are well-educated and health conscious, seeking medical attention more often. This group is followed by 30-44 years $(17.1 \%)$. Both age groups make up the active working population who are at higher risk of exposure to tropical infections. Similar findings have also been reported in studies by Chitkara et al, (2018) where the majority of cases, $68.3 \%$ were males and $31.7 \%$ were females, with most patients being in the productive phase of life (15-45 years) ${ }^{20}$. In another study, the age groups commonly affected were between $11-40$ years (68\%) witha higher number of cases, $56.3 \%$ skewed towards males while the other $43.7 \%$ comprising off emales $^{21}$. Males are associated with more outdoor activities leading to easy exposure to mosquito bite during the day time ${ }^{22}$. In addition, greater access to health care facilities and early diagnostic evaluation contribute towards a male predominance in dengue detection.

Most dengue patients detected during this study were males $(68.6 \%)$, although male patients were also more likely to be enrolled in the study (69.3\%). This difference likely arose from unequal health care utilization among male and female patients, which is a typical feature in South Asia ${ }^{23}$. The proportion of male febrile patients (38.52\%), who were positive for dengue was similar to that of female febrile patients $(39.85 \%)$, with the difference not being statistically significant $(P>0.05)$. Similarly, in another study conducted within six tertiarylevel referral teaching hospitals in Bangladesh, the proportion of female febrile patients positive for dengue was at just $8 \%$ compared to male participants at $11 \%$, the difference once again lacking statistical significance $(P>0.2)^{24}$. In a further study conducted in Khulna Medical College Hospital in southern Bangladesh, they found that among the enrolled dengue patients of paediatric group, no statistical sex difference occurred: 62 boys (53.9\%) compared with 53 girls $(46.1 \%)^{25}$. Additionally, there was no significant sex difference in either a study in Thailand with male and female infants (male: female ratio $=1.3: 1$ ), or in studies in Brazil and Peru with older patients ${ }^{26,27,28}$.

The clinical profile of dengue cases revealed that the most common presenting symptom was fever $(100 \%)$ followed by headache $(61.14 \%)$, Myalgia (44\%) and arthralgia (23.14\%). Retroorbital pain $(20.29 \%)$, a cardinal feature of dengue fever, was seen in a small subset of our patients $(20.29 \%)$. In the present study gastrointestinal symptoms including vomiting and abdominal pain $(32.86 \%)$ and loose motion $(5.14 \%)$ were also observed due to the liver injury caused by the dengue virus. Lower-back pain $(3.14 \%)$, rash $(6 \%)$ and conjunctival congestion were also seen less frequently as compared to other studies. Most of the patients presented with classical dengue, while dengue haemorrhagic fever and dengue shock syndrome occurred in a minority group. Similar studies in and around Bangladesh have also demonstrated fever as being the most prevailing symptom presented. A study by Kashinkunti et al, (2013) found fever as the most common presentation $(100 \%)$, followed by headache $(90 \%)$, myalgia $(81 \%)$, vomiting $(56 \%)$ and abdominal pain (48\%). Frequent clinical features including fever $(100 \%)$, headache (91\%), myalgia/arthralgia (85\%), vomiting (64\%), macular rash (55\%) and bleeding manifestation (46\%) (melena and bleeding gums) as found in another study ${ }^{29}$.

The temporal distribution showed that the dengue infection occurred throughout the year with a double peak persisting for 8 months, from May to December (pre-monsoon, monsoon and post-monsoon). In the previous years, dengue cases started to decline by September. However, in 2018 dengue incidences persisted through December. Surprisingly, the highest number of 
cases were found in the month of November. In short, dengue cases were detected year-round with sustained transmission, similar to other dengue endemic countries of Southeast Asia ${ }^{19}$. Climate change i.e. Rainfall throughout the year provides optimal conditions for breeding and egg hatching of Aedes aegypti vector. Moreover, mosquito survival, reproduction and virus transmission are influenced by temperature. Studies have shown, a rise of temperature from 17 to 30 Ú $\mathrm{C}$ increases dengue transmission fourfold ${ }^{19}$. In 2018, cases more than tripled in the capital, driven by a rapidly growing population, unplanned rapid urbanisation leading to considerable construction sites, contributing to the favourable conditions for the mosquitoes to breed as well as poor solid management. Eggs of the Aedes egyptiae can survive in such solid areas for more than 2 years $^{19}$.

Lastly, dengue epidemics can be significantly reduced if more pragmatic and well maintained urban-planning and improved drainage systems were fully implemented in cities. More awareness programs should additionally be carried out to minimize dengue infection by informing the general public precisely the means to prevent the availability of Aedes mosquito breeding-grounds in their local capacity. Moreover, females should get priority to access to treatment as they may be the silent source of infection.

\section{References:}

1. World Health Organization Bangladesh. Communicable Diseases. Available at: http:// www.whoban.org/communicable_dis.html.

2. Rahman M, Rahman K, Siddque AK, Shoma S, Kamal AHM, Ali KS et al (2002). First outbreak of dengue hemorrhagic fever, Bangladesh. Emerg Infect Dis. 2002; 8(7):738-740. doi:10.3201/eid0807.010398.

3. Guzman MG, et al (2010);. Dengue: a continuing global threat. Nat Rev Microbial. 2010; 8(12 Suppl):S716.

4. Simmons CP, et al (2012). Dengue. N Engl J Med.; 366(15):1423-32.

5. Halstead SB(1989). Antibody, macrophages, dengue virus infection, shock, and hemorrhage: a pathogenetic cascade. Rev Infect Dis.; 11 (Supplement 4):S830-9.
6. Nepal HP, Ansari S, Gyawali N, Gautam R, Paudel R, et al. (2014). Detection of IgM against Dengue Virus in Clinically Suspected Patients Presenting at a TertiaryCare Centre, Narayani Zone, Nepal. J Trop Dis 2: 139. doi:10.4172/2329-891X.1000139.

7. Halstead SB(2007). Dengue. The Lancet. ;370(9599):1644-52.

8. World Health Organization. Dengue Hemorrhagic Fever: Diagnosis, Treatment, Prevention and Control. Second edition. Geneva: World Health Organization, 1997: 1-84.

9. Sharmin S, Viennet E, Glass Kand and Harley D. The emergence of dengue in Bangladesh: epidemiology, challenges, and future disease risk. Trans R Soc Trop Med Hyg. 2015;109(10): 1-9.

10. Morales I, Salje H, Saha S, and Gurley ES. Seasonal Distribution and Climatic Correlates of Dengue Disease in Dhaka, Bangladesh. Am. J. Trop. Med. Hyg. 2016; 94(6), 359-1361.

11. Aziz MA, Gorham JR and Gregg MB. "Dacca Fever"An outbreak of dengue. Pakistan Journal of Medical Research. 1967; 6: 83-92.

12. Yunus EB, Bangali AM, Mahmood MAH, Rahman MM, Chowdhury AR and Talukder RK. Dengue outbreak 2000 in Bangladesh: From speculation to reality and exercise. Dengue Bulletin. 2001; 25: 1520.

13. Amin MMM, Hussain AMZ, Nahar K, Chowdhury IA, Murshed M and Chowdhury SA. Sero-diagnosis of dengue infections in four metropolitan cities of Bangladesh. Dengue Bulletin. 2000; 24: 34-41.

14. Pervin M, Tabassum S, Ali MM, Mamun KZ and Islam MN. Clinical and Laboratory Observations associated with the 2000 Dengue Outbreak in Dhaka, Bangladesh. Dengue Bulletin. 2004; 28: 96-106.

15. Sultana N, Biswas SK, Sultan T, Ahmed S,Hossain Z, Chowdhury R. Seroprevalence of dengue fever in Chittagong, Bangladesh. Chatt Maa Shi Hosp Med Coll J. 2013; 12(1): 38-40.

16. Sharmin R, Tabassum S, Mamun KZ, NessaA and Jahan M. Dengue infection in Dhaka City, Bangladesh. Mymensing Medical College Journal. 2013; 22(4):781-786.

17. Dhar-Chowdhury P, Paul KK, Haque CE, Hossain S, Lindsay LR, Dibernardo A, Brooks AW, Drebot MA. Dengue seroprevalence, seroconversion and risk factors in Dhaka, Bangladesh. PLoS Negl Trop Dis. 2017;11(3): e0005475. https://doi.org/10.1371/ journal.

18. Shirin T, Muraduzzaman AKM, Alam AN, Sultana S et. al Largest dengue outbreak of the decade with high fatality may be due to reemergence of DEN-3 serotype in Dhaka, Bangladesh, necessitating immediate public health attention. New Microbes New Inf. 2019; 29: 100511doi: 10.1016/ j.nmni.2019.01.007. 
19. Muraduzzaman AKM , Alam NA, Sultana S et.al. Circulating dengue virus serotypes in Bangladesh fronm 2013 to 2016. VirusDisease.2018; 29(3), pp 303-307.

20. Chitkara, S, Chhina, D , Gupta, V, Mahajan, R , Sharma, D . (2018). Epidemiology of Dengue Fever among clinically Suspected Febrile Patients at A Tertiary Care Center in Punjab. Journal of Microbiology and Infectious Diseases, 08 (02), 43-48. DOI: $10.5799 /$ jmid.434590

21. Chrispal A, Boorugu H, Gopinath KG, et al. Acute undifferentiated febrile illness in adult hospitalized patients: the disease spectrum and diagnostic predictors - an experience from a tertiary care hospital in South India. Trop Doctor 2010; 40(4):230234.

22. Kashinkunti M, Shiddappa, Dhananjaya MA (2013). Study of Clinical Profile of Dengue Fever in a Tertiary Care Teaching Hospital. Sch J App Med Sci.; 1(4):2802 .

23. Chajhlana SPS, Mahabhasyam RN, Varaprasada MSM, Anukolu RSR. Socio demographic and clinical profile of dengue fever cases at a tertiary care hospital, Hyderabad, Telangana. Int J Community Med Public Health 2017;4:2027-30.
24. Guha-Sapir D, Schimmer B, (2005) . Dengue fever: new paradigms for a changing epidemiology. Emerg Themes Epidemiol 2:1.

25. Yunus EB, Banu D, Talukder KR, Chowdhury MJ, Bangali AM, Montanari RM, 2002. Seroepidemiological study of dengue/ dengue haemorrhagic fever in a metropolitan hospital in Bangladesh. Dengue Bull 26: $1-6$.

26. Rasul CH, Ahasan HA, Rasid AK, Khan MR, (2002). Epidemiological factors of dengue hemorrhagic fever in Bangladesh. Indian Pediatr 39: 369 - 372.

27. Hung NT, Lan NT, Lei H , Lin Y, Lien LB, Huang K, Lin C, Ha DQ, Huong VTQ, My LT, Yeh TM, Huang J, Liu C, Halstead SB, (2005) . Association between sex, nutritional status, severity of dengue hemorrhagic fever, and immune status in infants with dengue hemorrhagic fever. Am J Trop Med Hyg 72: $370-374$.

28. Neto VSG, Rebêlo JMM , (2004). Epidemiological characteristics of dengue in the Municipality of São Luís, Maranhão, Brazil, 1997-2002. Cad Saude Publica 20: $1424-1431$.

29. Reiskind MH, Baisley KJ, Calampa C, Sharp TW, Watts DM, Wilson ML, (2001). Epidemiological and ecological characteristics of past dengue virus infection in Santa Clara, Peru. Trop Med Int Health 6: $212-218$. 